\title{
Experiência de Implantação do MR-MPS-SV no Service Desk da ECO Sistemas
}

\author{
Larissa Lopes de Araujo ${ }^{1}$, Elizabeth Cristina Mocny ${ }^{1}$, \\ Ana Regina Rocha ${ }^{3,4}$, Taísa Gonçalves ${ }^{3,4}$, Gleison Santos ${ }^{2}$ \\ ${ }^{1}$ ECO Sistemas \\ Rua Dom Gerardo, no 35 - $9^{\circ}$ andar - CEP 20.090-030 - Rio de Janeiro - RJ - Brasil \\ ${ }^{2}$ Programa de Pós-Graduação em Informática - UNIRIO \\ Av. Pasteur 458, Urca - CEP 22290-240 - Rio de Janeiro - RJ - Brasil \\ ${ }^{3}$ Programa de Engenharia de Sistemas e Computação - COPPE/UFRJ \\ Caixa Postal 68.511 - CEP 21.945-970 - Rio de Janeiro - RJ - Brasil \\ ${ }^{4}$ Implementum - Instituição Implementadora MPS \\ \{larissa.araujo; elizabeth.mocny\}@ecosistemas.com.br; \\ \{darocha, taisa\}@cos.ufrj.br; gleison.santos@uniriotec.br
}

\begin{abstract}
This paper describes the experience of ECO Systems with the implementation of the MR-MPS-SV Level G in its Service Desk department. The implementation began in September 2013 and was completed with the official appraisal in December 2013. Results indicate an increase in productivity related to providing the service, visibility of SLA demanded, and the construction of historical data.
\end{abstract}

Resumo. Este artigo descreve a experiência da ECO Sistemas com a implementação do nível G MR-MPS-SV no seu Service Desk. A implementação teve inicio em Setembro de 2013 e a avaliação oficial foi concluída em Dezembro de 2013. Os resultados obtidos indicam aumento de produtividade na prestação do serviço e visibilidade de SLA atendida, além da criação de base histórica.

\section{Introdução}

A competição do mercado de Tecnologia da Informação leva as organizações a optarem em implementar cada vez mais modelos de melhoria de processos de software e serviços, com o objetivo de estarem aptas a conquistar e manter sua participação neste marketshare [PARDO et al. 2012]. Estudos recentes [PARDO et al. 2011] apontam o crescimento das publicações que apresentam a harmonização de modelos e normas de qualidade em diversas disciplinas, como desenvolvimento, manutenção, operação, segurança e governança de TI. Conforme Pardo et al. (2011), seria imprudente acreditar que qualquer modelo definido até o presente momento possui uma solução completa para o gerenciamento da Tecnologia da Informação. Esta constatação levam muitas organizações a optarem pela melhoria de seus processos utilizando múltiplos modelos. Porém, quando uma iniciativa de melhoria de processo de software multimodelos não é 
implantada adequadamente, pode acarretar esforço e custo adicionais, assim como riscos de ineficiências e redundâncias [Furtado et al. 2012].

Com 23 anos de atuação no segmento da Saúde Pública, a ECO Sistemas é uma empresa de Tecnologia da Informação que adota múltiplos modelos pertinentes ao seu negócio. Já conta hoje com alguns modelos e normas implantados e integrados, como o modelo de gerenciamento da melhoria da qualidade ISO 9001:2008, o modelo para melhoria de processos de software MR-MPS-SW Nível C, o modelo de melhoria do processo de testes de software MPT Nível 1 [SOFTEX RECIFE 2011].

Com um recente setor de Service Desk criado, a ECO percebeu a necessidade de aplicar um novo modelo. Esse setor realiza atendimento ao cliente, em especial na manutenção dos serviços de software e correlatos. De início, como nenhum modelo anteriormente adotado pela organização era adequado a esse fim, utilizou-se processos já implantados na Empresa para organizar a forma de trabalho. No entanto, ainda não era o adequado.

O modelo MPS para Serviços, o MR-MPS-SV [SOFTEX, 2012], possui o objetivo de apoiar as organizações na implantação de boas práticas para gerenciamento de serviços de TI. Mesmo possuindo um grupo de processos e uma larga experiência na implantação de melhoria de processos, a ECO Sistemas decidiu contratar uma consultoria especializada com experiência na implantação do MR-MPS-SV. Desta forma, em agosto de 2013 foi contratada a IMPLEMENTUM, Instituição Implementadora MPS credenciada para o modelo MR-MPS-SV.

O objetivo deste artigo é apresentar como a ECO Sistemas foi a primeira organização a implantar o MR-MPS-SV (excluindo os projetos pilotos) em tempo recorde (quatro meses) que culminou com a conquista do nível $\mathrm{G}$ em dezembro de 2013. Além dessa introdução, o artigo está dividido nas seguintes seções: a Seção 2 apresentado o modelo MR-MPS-SV; a seção 3 apresenta experiências anteriores de melhorias de processos na ECO Sistemas e seu histórico de busca da qualidade; a Seção 4 apresenta a implantação do MR-MPS-SV, descrevendo etapas e prazos, desafios e resultados; por fim, a Seção 5 apresenta as considerações finais.

\section{O Modelo MR-MPS-SV}

O MR-MPS-SV, publicado em agosto de 2012, possui como base técnica a IEC/IEC 20000:2011 [ISO/IEC, 2011], a ISO/IEC 15504-2:2003 [ISO/IEC, 2003] e CMMISV:2009 [CMMI Product Team 2010b]. Em pesquisa recente, 95\% dos implementadores do MR-MPS-SW consultados concordam que a implantação do MRMPS-SV em organizações que já utilizam o MR-MPS-SW traz benefícios de qualidade e produtividade [JORDÃO et al. 2013].

Com 24 processos distribuídos nos 7 níveis de maturidade, o MR-MPS-SV proporciona a implantação continua de boas práticas para gerência de serviços de TI. Desta forma, o nível G (Parcialmente Gerenciado) contempla os processos Entrega de Serviço, Gerência de Incidentes, Gerência de Nível de Serviço, Gerência de Requisitos e Gerência de Trabalho. O nível F (Gerenciado) inclui os processos Aquisição, Gerência de Configuração, Garantia da Qualidade, Gerência de Problema, Gerência de Portfólio do Trabalhos e Medição. Para atingir o nível E (Parcialmente Definido) a organização 
deve implementar, além dos anteriores, os processos Avaliação e Melhoria do Processo Organizacional, Definição do Processo Organizacional, Gerência de Mudança, Gerência de Recurso Humanos e uma evolução do processo Gerência de Trabalho. No nível D (Largamente Definido) são incluídos os processos Desenvolvimento do Sistema de Serviços, Orçamento e Contabilização de Serviços e no nível C (Definido), Gerência de Capacidade, Gerência da Continuidade e Disponibilidade dos Serviços, Gerência de Decisões, Gerência de Liberação, Gerência de Riscos, Gerência da Segurança da Informação e Relato do Serviço. No nível B (Gerenciado Quantitativamente) deve ser implementada uma nova evolução da Gerência do Trabalho. No nível A (Em Otimização) não são incluídos novos processos, pois este nível se caracteriza apenas pela evolução dos atributos do processo.

\section{Experiências Anteriores de Melhorias de Processo na ECO Sistemas}

A implantação de modelos de qualidade em uma organização envolve bastante conhecimento. A equipe de gestão da qualidade precisa investir em informação e contar com a ajuda de especialistas na implantação dos modelos de qualidade, para evitar as redundâncias e esforços extras [FURTADO et al. 2012].

Em 2002, a ECO Sistemas iniciou sua escalada rumo à melhoria da qualidade de seus processos e produtos, por meio da obtenção da certificação ISO 9001:2008 para todo o seu escopo de trabalho. Instituiu seu Grupo Gestor da Qualidade e o Sistema de Gestão da Qualidade. Os 14 processos iniciais da empresa construíram a base que seria utilizada após, nas iniciativas de implantação de modelos, como a Produção de Documentos, Registros da Qualidade, Tratamento de Processos e Produtos NãoConformes, Ações de Melhorias, Preventivas e Corretivas, Treinamento, Compras, Recursos Humanos e Infraestrutura. Em 2008, iniciou a implantação de um novo modelo mais específico para seu negócio principal, o MR-MPS-SW Nível F.

Os processos Gerência de Projetos e Gerência de Requisitos foram devidamente integrados ao processo de desenvolvimento de software. Os processos de Garantia da Qualidade, Medição e Gerência de Configuração deram origens a novos procedimentos operacionais e novas práticas [ARAUJO et al., 2010]. Através desta experiência, a ECO Sistemas formou uma equipe de cinco PMPs (Project Management Professional) e implantou uma ferramenta da Microsoft de gestão do ciclo de vida do software, o TFS Team Foundation Server. Dois meses após a avaliação MPS nível F, a empresa harmonizou seus processos com as práticas do CMMI-DEV [CMMI Product Team, 2010a] e em dezembro de 2009 foi avaliada no CMMI Nível 2.

Com o objetivo de melhoria contínua, após um ano, a ECO tinha uma nova meta: implantar o Nível C do MR-MPS-SW. Este projeto contou com apoio de consultoria, com o esforço de toda a equipe de desenvolvimento e com a equipe da qualidade. A avaliação oficial MPS Nível C foi obtida no final de 2012.

Em julho de 2013, a ECO Sistemas percebeu as novas tendências e iniciou seu novo desafio: implantar simultaneamente três novos modelos: O MPT para melhoria do processo de teste [SOFTEX RECIFE 2011], o CERTICS [CTI Renato Archer 2013] e o MR-MPS-SV. Neste artigo apresentamos, apenas, o processo de implantação do MRMPS-SV. 


\section{Implantação do MR-MPS-SV}

A ECO Sistemas vislumbrou a implantação do nascente MR-MPS-SV como uma oportunidade de para atingir as melhores práticas na gerência de serviços e vir a ser referência no mercado para sistemas voltados para a área de saúde.

\subsection{Etapas e Prazos}

A consultoria contratada possuía grande experiência em implementações do modelo MR-MPS-SW. No início do projeto, em Setembro de 2013, foram identificadas cinco etapas importantes do processo:

- Análise da situação atual do processo da equipe do Service Desk. (Setembro/2013). Nesta etapa a consultoria analisou o processo em uso do Service Desk, sua equipe e sua ferramenta OTRS (Open Tecnology Real Service). Uma análise de viabilidade foi realizada na OTRS e identificou-se que é uma ferramenta gratuita de Gerência de Serviços que disponibiliza 3 níveis de atendimento, com a aplicação de alertas por ANS/SLA e configuração de Serviços. As funcionalidades não eram utilizadas. A configuração dos serviços por cliente define o escopo a ser contemplado pelo projeto, restringindo as opções na abertura do tíquete.

- Revisão e realização das adequações necessárias ao processo. (Outubro/2013). O objetivo foi implantar os processos Gerência de Trabalho e Gerência de Requisitos para entender o requisito do contrato e sua gestão. Os processo Gerência de Nível de Serviço, Gerência de Incidentes e Entrega de Serviço foram apoiados diretamente pela ferramenta OTRS. O ANS/SLA foi configurado na OTRS, por cliente (funcionalidade existente no software), para gerir as prioridades. As atividade que atingiam o ANS/SLA tratado emitiam alertas. Os incidentes recebidos eram classificados como tal, para seu tratamento. Os scripts de tratamentos de incidentes previstos foram registrados na base de conhecimento da OTRS (funcionalidade existente no software). A Entrega de Serviços foi tratada pela ferramenta, pois mensalmente era extraído os chamados atendidos, para o fechamento do Relatório Técnico Mensal, entregue ao Cliente como atestado de trabalho realizado. Além dos processos do Nível G do MR-MPS-SV, optou-se por implementar o processo Garantia da Qualidade (presente no Nível F), por sua importância para verificação da aderência aos processos e da qualidade dos produtos de trabalho.

- Capacitação da equipe nos novos processos. (Novembro /2013). Após definido o processo de Service Desk, a equipe foi capacitada no Processo de Service Desk e nas configurações da ferramenta OTRS.

- Implantação do novo processo. (Novembro/2013). O início da utilização do processo não teve grandes impactos, resultado da experiência anterior nas tarefas, dos treinamentos e mentoring.

- Avaliação formal MR-MPS-SV Nível G. (Dezembro/2013). Nesta fase foi preenchida a planilha de indicadores, contratada a avaliadora e realizada a avaliação avaliação oficial MR-MPS-SV. 
O primeiro mês foi utilizado pela equipe de consultores para entender o processo interno, mapear as necessidades da organização e conhecer a ferramenta OTRS.

No segundo mês, a equipe da qualidade atuou com o gerente do Service Desk e a consultoria na definição de novos processos aderentes ao MR-MPS-SV e com as adaptações da OTRS. Nesta ferramenta foi possível implementar: ANS (Acordo do Nível de Serviço, ou SLA, da sigla em inglês para Service Level Agreement) por cliente, o Catálogo de Serviços por cliente, a diferenciação no registro de incidentes, a base de conhecimento por problemas repetitivos, assim como a distinção de incidentes no cliente e incidentes internos. Os requisitos de cada trabalho incluem o ANS/SLA definido pelo cliente. $\mathrm{O}$ gerente do setor assumiu a responsabilidade de acompanhar os Acordos de Nível de Serviço (ANS/SLA) com o monitoramento do tempo gasto para sua execução dos tíquetes dos atendimentos em $1^{\circ}$ e $2^{\circ}$ níveis com reports mensais.

No terceiro mês foi realizada a capacitação da equipe de Service Desk em etapas, visto que a equipe trabalha em escala, de $24 \times 7$. Para essa capacitação, o mentoring on the job demonstrou ser bem eficaz.

O quarto mês foi utilizado para preparar a empresa para a avaliação: contratação da Instituição Avaliadora, preparação da planilha de indicadores, definição da logística da avaliação e realizaçãoda avaliação. Em dezembro de 2013 a ECO Sistemas foi avaliada MR-MPS-SV e conquistou o nível $\mathrm{G}$ com inclusão do processo Garantia da qualidade, após avaliação com mais de 20 colaboradores entrevistados em 2 dias, entre a avaliação inicial e final.

\subsection{Desafios}

Inicialmente os maiores obstáculos seriam, sob o ponto de vista da equipe da qualidade da empresa, o baixo nível de conhecimento da equipe de Service Desk, o prazo ousado de 4 meses para a implementação e avaliação, a ferramenta OTRS não dar suporte a todas as exigências do modelo e possível dificuldade de obter envolvimento do gerente do setor e de sua equipe.

Além disso, não se pode esquecer que a empresa também estava envolvida na implantação de dois outros modelos (CERTICS e MPT) com outras consultorias.

\subsection{Resultados}

Algumas características da organização contribuíram para o tempo de implantação reduzidos, como a existência de um Service Desk em funcionamento com ferramenta já difundida, como um coordenador especialista com o conhecimento do modelo MRMPS-SV (Nível C) e equipe de definição de processo dedicada (Gerência da Qualidade).

Os dados da atuação do Service Desk demonstram que, após a implantação do modelo, houve de fato ganho de produtividade. Com quase um ano de atuação a equipe já recebeu 6.509 chamados de atendimento. A Figura 1 apresenta a evolução do atendimento da equipe por mês deste sua instituição, em junho de 2013, passando pelos meses de implantação do MR-MPS-SV, de setembro à dezembro de 2013 (de 9-12) até abril de 2014. Como a equipe se manteve com o mesmo tamanho, é possível concluir que a implantação da MR-MPS-SV trouxe ganhos de produtividade da equipe de 7,06\% 
(a diferença entre média de produtividade após a implantação, de outubro/2013 à abril/2014 [78,86\%] com a produtividade média anterior, de junho/2013 à setembro de 2013 , de $[71,80 \%])$.

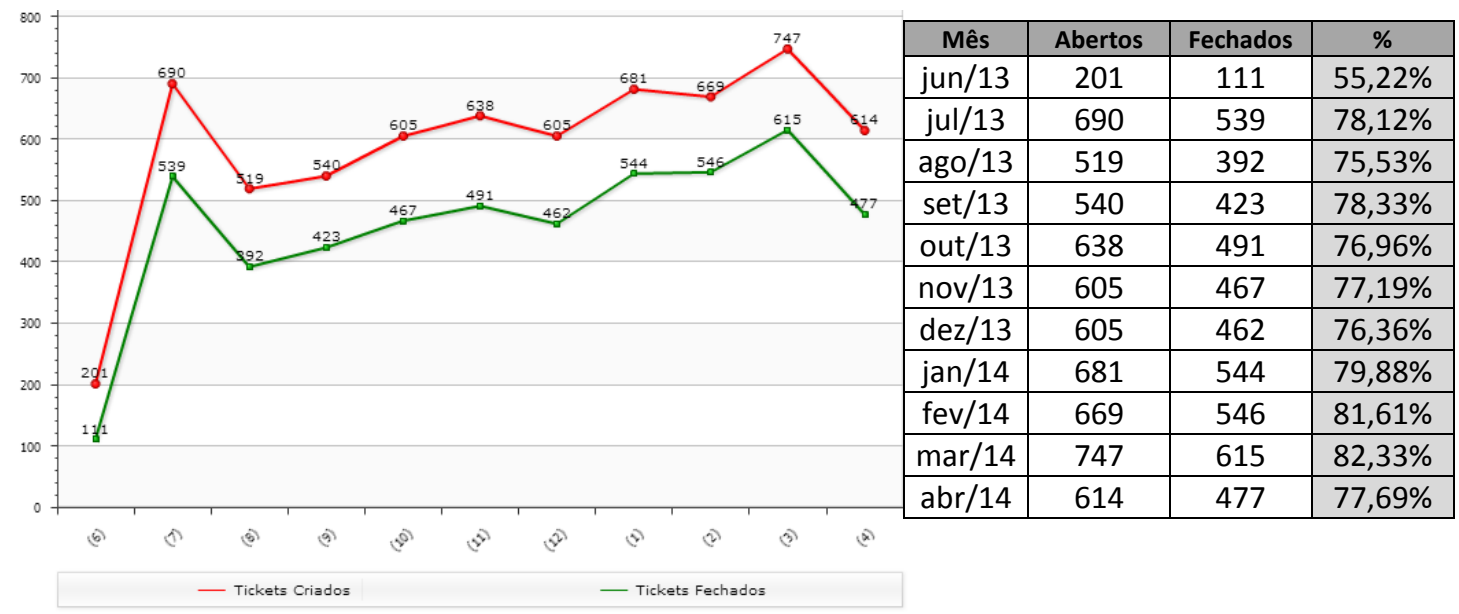

Figura 1. BI de tíquetes do OTRS de junho/2013 à abril/2014

Outro ponto observado é a redução do tempo de resposta ao cliente. Na Figura 2 pode-se observar uma redução em relação ao Tempo de Resposta (indicador que apresenta o tempo entre a abertura do tíquete pelo cliente e a primeira resposta do atendente Service Desk). Este indicador apresenta uma redução de 88,91\% (de 5.299 minutos em outubro/2013 à 417 minutos em abril/2014). Além desse, o segundo indicador apresentado na Figura 2 é o Tempo de Solução (indicador que apresenta o tempo entre a abertura do tíquete pelo cliente e a solução dada pela equipe). Este indicador apresenta uma redução de $92,13 \%$ (de 13.420 minutos em outubro/2013 à 1486 minutos em abril/2014).

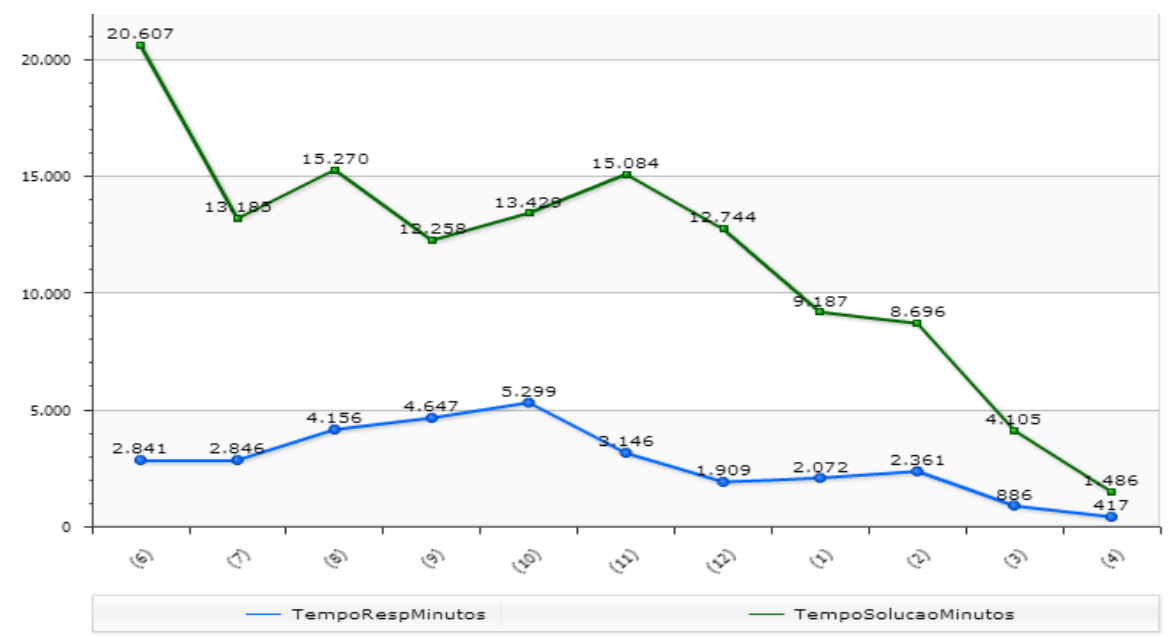

Figura 2. BI de Tempo de Resposta aos Tíquetes de Junho/2013 à Abril/2014

Ao observar os resultados de produção da equipe Service Desk ao longo dos onze meses de atuação verifica-se que vários fatores contribuíram para o aumento do desempenho, porém todos os fatores possuem referência com o processo implantado. 


\section{Considerações Finais}

Os principais benefícios obtidos com a experiência na implantação do primeiro nível de maturidade do MR-MPS-SV foi constatar que para atender o cliente em serviços de manutenção continuada é preciso entender seus requisitos e planejar o trabalho. Este planejamento deve ser de conhecimento de todos os membros da equipe, assim como seus requisitos do trabalho e respectivos ANSs/SLAs.

Durante a elaboração dos Planos de Trabalho com o levantamento dos contratos, foi possível avaliar que o gerente do Service Desk não possuía, em alguns momentos, conhecimento de todo o escopo do contrato(ou pior: acreditava ser maior o requisito do cliente). Desta forma é possível afirmar que a falta de gestão do plano de trabalho do serviço e o desconhecimento de parte dos requisitos levam ao atendimento de serviços não contemplados pelo cliente. E isto é muito comum, pois a equipe de Service Desk simplesmente atende aos chamados dos clientes, sem questionar se é ou não do escopo. A configuração do requisito do cliente na ferramenta (com a seleção do catálogo por cliente) e seu ANS/SLA contratado promoveu o aumento dos indicadores "Tempo de resposta" e "Tempo de Atendimento". Desta forma, considerando do mês de implantação do MR-MPS-SV (Outubro/2013) até abril/2014, houve uma redução substancial dos indocadores de tempo de resposta e atendimento, de $88,91 \%$ e $92,13 \%$ respectivamente, demonstrando melhoria de produtividade. Logo pode-se afirmar que a adequada implantação dos processos Gerência do Trabalho e Gerência de Requisitos, no âmbito do MR-MPS-SV, reduz desperdícios da equipe de Service Desk e aumenta a sua produtividade.

Outro aspecto observado na implantação foi o aumento da efetividade da equipe, ao realizar a gestão por ANS/SLA. Este fator se deve à cobrança mais focada do gerente do setor na priorização das tarefas para o atendimento da ANS/SLA. O envio de alertas de acordos de níveis de serviços através de uma ferramenta possibilita a correção de desvios em sua ocorrência. Logo, a implantação da gestão por ANS/SLA reduziu em 7,06\% o tempo médio de atendimento dos pedidos de serviços.

O baixo nível de conhecimento da equipe de Service Desk foi apoiada pela identificação das necessidades de treinamentos específicos, requisito do trabalho organizacional, gerando motivação e conhecimento reforçado aos colaboradores.

A configuração da base de conhecimento da ferramenta OTRS apoiou a equipe, normalmente formada por um nível de formação mais simples. Esta base cadastra um tratamento a um problema específico para que seja utilizado como fonte de tratamento de problemas similares, formando, assim, uma base para apoiar o operador.

O investimento realizado na contratação de consultoria, equipe de processos, avaliação interna e externa e logística ficou em torno de $\mathrm{R} \$ 70.000,00$. Uma análise em relação ao retorno do investimento foi realizada. Se o custo da equipe é cerca de $\mathrm{R} \$ 40.000,00 /$ mês, e houve de fato uma economia de no mínimo 7,06\% de trabalho, é possível afirmar que a equipe produziu $7,06 \%$ a mais. Logo, a Empresa ganhou o equivalente $\mathrm{R} \$ 2.824,00$ a mais em forma de trabalho por mês. Desta forma, considerando o custo do projeto, este se paga em de 25 meses.

Os próximos passos da equipe de Qualidade da ECO Sistemas para o Setor de Service Desk é o crescimento contínuo dos níveis de maturidade, para que se possa 
absorver novos processos considerados importante pelo modelos MR-MPS-SV. Outro ponto observado por esta equipe é a necessidade de integração da ferramenta OTRS (de gestão de tíquetes de serviços) com a ferramenta TFS (de ciclo de vida do software).

Por fim, é importante reforçar que os modelos de qualidade existentes podem e devem ser harmonizados para valorizar e organizar as diversas equipes dentro da organização de forma que todos possam se enxergar como parte importante do todo e garantir que a implantação dos modelos vai ser benéfica, de fato, para a empresa e não apenas para seguir o modelo simplesmente.

\section{Referência}

ARAUJO, L. L. e MELLO, A. B. 2010. Implantação do MPS.BR (Melhoria do Processo de Software Brasileiro), nível F, com TFS (Team Foundation Server) no Desenvolvimento Eficiente de Sistemas. WAMPS - Workshop Anual do MPS. 2010.

CMMI Product Team. 2010a. CMMI for Development, Version 1.3. Pittsburgh, Pennsylvania : Software Engineering Institute, Carnegie Mellon University, 2010a. Technical Report CMU/SEI-2010-TR033.

CMMI Product Team. 2010b. CMMI for Services, Version 1.3. Pittsburgh, Pennsylvania : Software Engineering Institute, Carnegie Mellon University, 2010b. CMU/SEI-2010-TR-034.

CTI Renato Archer. 2013. Modelo de Referência para Avaliação da CERTICS - Documento de Detalhamento. Campinas: Centro de Tecnologia da Informação Renato Archer, 2013.

FURTADO, J. C. e OLIVEIRA, S. R. B. 2012. A Process Framework for the Software and Related Services Acquisition Based on the CMMI-ACQ and the MPS.BR Acquisition Guide. IEEE Latin America Transational, Vol. 10, nº6, December 2012.

ISO/IEC. INTERNATIONAL ORGANIZATION FOR STANDARDIZATION/ INTERNATIONAL ELETROTECHNICAL COMISSION. 2008. ISO/IEC 12207 Systems and Software Engineering Software Life Cicle Process. Geneve, 2008.

ISO/IEC. INTERNATIONAL ORGANIZATION FOR STANDARDIZATION/ INTERNATIONAL ELETROTECHNICAL COMISSION. 2011. ISO/IEC 20000 Information Technology - Service Management. Geneve, 2011.

ISO/IEC. INTERNATIONAL ORGANIZATION FOR STANDARDIZATION/ INTERNATIONAL ELETROTECHNICAL COMISSION. 2003. ISO/IEC 15504-2: Information Technology - Process Assessment - Part 1: Concepts and Vocabulary. Geneve, 2003.

JORDÃO, L. e KALINOWSKI, M. 2013. Investigando a aplicabilidade do MPS-SV na Melhoria de

Serviço de Desenvolvimento e Manutenção de Software. WAMPS - Workshop Anual do MPS. 2013.

OTRS - Open Tecnology Real Service. 2014. OTRS - Open Tecnology Real Service. [Online] 2014. [Citado em: 2014 de Abril de 25.] http://www.otrs.com.

PARDO, C.; PINO, F. J.; GARCÍA, F.; VELTHIUS, M. P. e BALDASSARE, M. T. 2011. Trends in Harmonization of Multiple Reference Models. L.A. Maciaszek and P. Loucopoulos (Eds.):ENASE 2010, CCIS 230, pp61-73. 2011.

RODRIGUES, LEONEL CEZAR. 2010. O Desenho da Gestão da Tecnologia da Informação nas 100 Maiores Empresas na Visão dos Executivos de TI. 2010.

SOFTEX. 2012. Guia Geral MPS de Serviços. 2012. Disponível em www.softex.br/mpsbr.

SOFTEX RECIFE. 2011. MPT.Br Melhoria de Processo de Teste Brasileiro - Guia de Referência do Modelo. 2011. 